\title{
Spacecraft Onboard Software Maintenance - An Effective Approach which Reduces Costs and Increases Science Return
}

\author{
Elaine Shell \\ National Aeronautics and Space Administration, Goddard Space Flight Center \\ Greenbelt, Maryland, U.S.A. \\ Yvonne Lue \\ Computer Sciences Corporation, Lanham, Maryland, U.S.A. \\ Martha I. Chu \\ The Johns Hopkins University, Applied Physics Laboratory, Laurel, Maryland,U.S.A.
}

\begin{abstract}
Flight software (FSW) is a mission critical element of spacecraft functionality and performance. When ground operations personnel interface to a spacecraft, they are dealing almost entirely with onboard software. This software, even more than ground/flight communications systems, is expected to perform perfectly at all times during all phases of on-orbit mission life. Due to the fact that FSW can be reconfigured and reprogrammed to accommodate new spacecraft conditions, the on-orbit FSW maintenance team is usually significantly responsible for the long-term success of a science mission. Failure of FSW can result in very expensive operations work-around costs and lost science opportunities.
\end{abstract}

There are three basic approaches to staffing on-orbit software maintenance, namely: (1) using the original developers, (2) using mission operations personnel, or (3) assembling a Center of Excellence for multi-spacecraft on-orbit FSW support. This paper explains a National Aeronautics and Space Administration, Goddard Space Flight Center (NASA/GSFC) experience related to the roles of on-orbit FSW maintenance personnel. It identifies the advantages and disadvantages of each of the three approaches to staffing the FSW roles, and demonstrates how a cost efficient on-orbit FSW Maintenance Center of Excellence can be established and maintained with significant return on the investment.

Keywords: flight software, flight software maintenance, operations

Abbreviations: CSC: Computer Sciences Corporation; CGRO: Compton Gamma Ray Observatory; FSW: flight software; FOT: flight operations team; IOC: In-orbit Checkout; NASAVGSFC: National Aeronautics and Space Administration, Goddard Space Flight Center; UARS: Upper Atmosphere Research Satellite; XTE: X-Ray Timing Explorer

\section{Introduction}

FSW distributes and executes large sequences of uplinked commands while at the same time, it:

- determines spacecraft attitude, orbit, momentum, power performance, etc.

- performs a variety of nominal and "safehold" control modes, plus manages momentum

- detects anomalies and responds in ways which protect flight hardware

- stores and plays back flight data

- generates telemetry and logs for full ground visibility into flight status and issues

- manages ground communications and processes ground commands.

With the ever-increasing capacities of space-qualified processors, FSW complexities are also increasing. Flight sensors, actuators and detectors are made more powerful and flexible via management of their functionality from onboard software. In addition, new onboard autonomy is called upon to reduce routine operations costs and to minimize the sizable science data rates.

1.1 Roles of On-orbit FSW Maintenance Staff.

Good FSW maintenance engineers are "working partners" with the Flight Operations Team (FOT). As such, they are routinely called upon to:

- explain FSW characteristics, including nominal responses to operations scenarios

- interpret unusual FSW statuses

- provide a range of support during on-orbit anomaly investigations

- develop and certify configuration changes to the FSW during safemode recovery

- analyze how FSW can be made to support new operations strategies

- develop and validate new/modified onboard health and safety monitors and limits 
- develop/validate new autonomous responses to onboard anomalies and failures

- help define requirements to change FSW or its data

- design, develop, test/validate, document, prepare for uplink, and certify on-orbit FSW changes In addition, the maintenance team ensures consistency of the FSW test environment to the on-orbit flight load contents and flight hardware characteristics.

\subsection{The FSW Test Facility.}

A good FSW test environment is the primary prerequisite for almost all on-orbit changes to FSW and its controlling parameters; as well as much of the on-orbit analysis of FSW behavior. Figure 1 represents a typical high fidelity FSW testbed at GSFC. The FSW is hosted into a computational environment, which accurately replicates the flight data system and its external interfaces. A closed-loop dynamic simulator accurately models the on-orbit environment of the spacecraft, accepts hardware commands from the FSW,

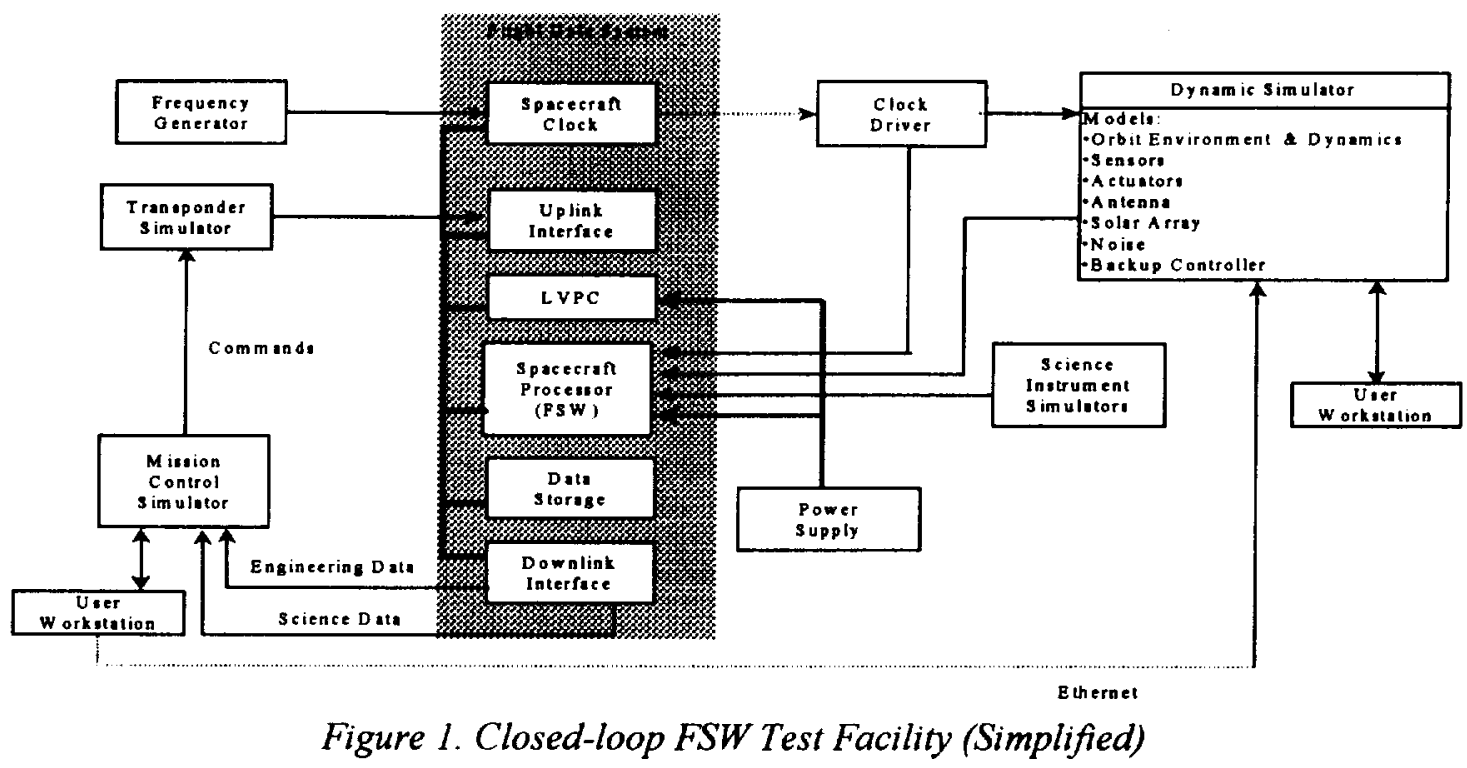

and generates appropriate hardware responses to the FSW. The dynamic simulator is fully configurable to exercise varied characteristics of the flight hardware and to inject errors into the flight system for purposes of testing FSW response to non-routine on-orbit events. A ground control system performs relevant mission control center functions using a command and telemetry database equivalent to the operations database.

\section{On-orbit Flight Software Maintenance Staffing Strategies}

The following subsections address three approaches of providing FSW skills to on-orbit missions. We believe the third approach, developing a team of FSW specialists who are fully dedicated to FSW support of multiple on-orbit missions, results in the best solution when considering mission risk, science productivity and cost.

\subsection{Use the Original FSW Development Staff.}

One common approach to FSW maintenance is to use the original development staff throughout the mission life. Since FSW is qualified prior to launch as being "error free", there is a tendency to believe that post-launch FSW support will be required only if an on-orbit anomaly occurs -- at which point the people who developed the FSW will be the most efficient people to resolve the on-orbit FSW issues. This approach has been used on occasion at the NASA/GSFC and at the Johns Hopkins University, Applied Physics Laboratory.

However, not only does this leave the FOT with minimal in-depth FSW support for the life of the mission, but if there is an on-orbit event, it can quickly become a challenge to obtain the services of these key people. If the developers were from the in-house organization, they will have been assigned to a different job, quite likely under different project management; and if the FSW development was done by an outside contractor, access to individuals can be even more difficult (especially as the on-orbit mission ages). If a developer hasn't worked on the FSW for awhile, he may need precious time to re-familiarize himself to the FSW, its testbed and tools; and a lack of insight 
into issues related to FSW in its on-orbit operations configuration may result in the developer being unprepared for the challenges.

FSW support for an on-orbit mission is really a different set of skills than FSW development of the initial load. It doesn't take many on-orbit experiences in this environment to discourage a developer from supporting such an activity again soon. In addition, most on-orbit FSW change requests are not due to FSW errors left by the developers. Most requests for on-orbit FSW changes result from:

- new insights into flight hardware characteristics,

- degradation or failure of flight hardware, or

- the desire for new control options to permit enhanced science capabilities.

An on-orbit event is often the start of significant activity to develop a long-term solution, via FSW, to resolve or prevent a recurrence of a problem. This is often when management recognizes the need for dedicated FSW staff.

As time passes and developer staff continues to move onto new jobs, the configuration control of the FSW development environment, the testbed, databases and tools may all be jeopardized. The longer the mission survives on-orbit, the more difficult and troublesome relying on development staff becomes. This approach is well described in the European Space Agency experiences (Maldari, et al. 1995), which recommends the next (2.2) approach.

\subsection{Assign FSW Staff to the FOT.}

The second approach is to train a member of the FOT to maintain the FSW. Because the FSW specialist is located with the FOT, a "seamless" interface between the two groups exists. Communications, delivery of products, and availability of in-depth FSW expertise to the FOT seems optimum.

This approach was used at GSFC for the Upper Atmosphere Research Satellite (UARS) mission, launched in 1990 and still very functional. The same contractor organization developing the spacecraft was also responsible for flight operations, making it appear to be especially cost effective to include FSW specialist staff within the FOT. Since the level of FSW work anticipated post-launch was an occasional patch, a single FSW specialist was trained for the on-orbit role. In the UARS case, the FSW specialist became extremely proficient in all aspects of on-orbit FSW support. Due to several flight hardware problems and selected FSW improvements, some exciting FSW work was accomplished. When not working on FSW issues, the FSW specialist served as attitude control subsystem analyst.

A disadvantage to this approach is that a very small FSW staff within the FOT can have a severe impact on the speed with which any new FSW activity can be accomplished. Also a lack of in-depth FSW peer review can be frustrating. In the UARS case, access to original spacecraft developers diminished dramatically after launch and was made available only during a single extreme crisis situation. Other persons brought in from outside organizations to support FSW reviews were either FSW specialists who were not knowledgeable of UARScharacteristics or they were subsystem specialists and not knowledgeable of detailed FSW issues. Over time, the isolation from other software engineers, combined with a lack of consistent and challenging FSW work, caused the UARS specialist to leave the FOT. Hiring a replacement for this unique role -- i.e., to support an obsolete flight computer and language within the FOT environment - can quickly prove to be a difficult task. Also, because there is little need for formalities within the close-knit FOT, the FSW process may not be well documented and available for new staff. With the very long learning curve associated with FSW, its testbed and tools, departure of small FSW staff can create extremely limited capability to support the FSW at all -- although significant science value from the mission continues. The next option (2.3) provides an answer to both the human and programmatic problems evident here.

2.3 Create an On-orbit FSW Center of Excellence. The third approach is to assemble a Center of Excellence for on-orbit FSW maintenance. In this case a team of software engineers is trained specifically to support FSW of multiple missions. The cost to any one mission is extremely low because the software staff works and charges minimally on missions with no current operations issues; yet multiple on-orbit FSW- experienced staff can be made available to a mission requiring FSW support. The professional insight into on-orbit FSW troubleshooting and maintenance methods ensures low risk, effective and minimal-cost solutions to on-orbit issues when they arise. The variety of challenges among a set of missions results in a dedicated, talented staff who are fully trained and available to support each mission's needs.

GSFC initiated an on-orbit FSW Center of Excellence during the late 1980s using Computer Sciences Corporation (CSC) as the support contractor. This single FSW team grew from a staff of 3 people supporting pre-launch preparations for one mission, to a current staff of 14.5 supporting 7 on-orbit missions and contributing to 3 missions under development. Because the team is contracted from the FSW organization at GSFC, FSW methods as well as resource needs are addressed with the full support of the larger government FSW experience base. Figure 
2 identifies the GSFC missions currently supported by this team; and the staff commitment per mission as a snapshot-in-time in January 1999. With 7 team members actively supporting missions not yet launched, the remaining 7.5 people are supporting the collection of missions on-orbit.

\section{On-orbit Missions:}

\begin{tabular}{|c|c|c|c|c|c|c|}
\hline $1 / 91$ & $6 / 92$ & $12 / 95$ & $6 / 96$ & $11 / 97$ & $3 / 98$ & $12 / 98$ \\
\hline $\begin{array}{c}\text { Compton } \\
\text { Gamma Ray } \\
\text { Observatory } \\
\text { (CGRO) }\end{array}$ & $\begin{array}{l}\text { Extreme } \\
\text { UItraVlolet } \\
\text { Explorer } \\
\text { (EUVE) }\end{array}$ & $\begin{array}{c}\text { X-Ray } \\
\text { Timing } \\
\text { Explorer } \\
\text { (XTE) }\end{array}$ & $\begin{array}{l}\text { Fast Auroral } \\
\text { Snapshop } \\
\text { Explorer } \\
\text { (FAST) }\end{array}$ & $\begin{array}{c}\text { Troplcal } \\
\text { Rainforest } \\
\text { Measurement } \\
\text { Mission (TRMM) }\end{array}$ & $\begin{array}{c}\text { Transition } \\
\text { Region and } \\
\text { Coronal Explorę } \\
\text { (TRACE) }\end{array}$ & $\begin{array}{c}\text { Submillimeter } \\
\text { Wave } \\
\text { Astronomy } \\
\text { Satellite } \\
\text { (SWAS) } \\
\end{array}$ \\
\hline 0.5 & 0.5 & 1.5 & 0.5 & 2 & 0.5 & 1.5 \\
\hline$L$ & 1 & 1 & 1 & $\perp$ & 1 & 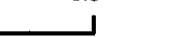 \\
\hline
\end{tabular}

Future Launches Currently Supported :
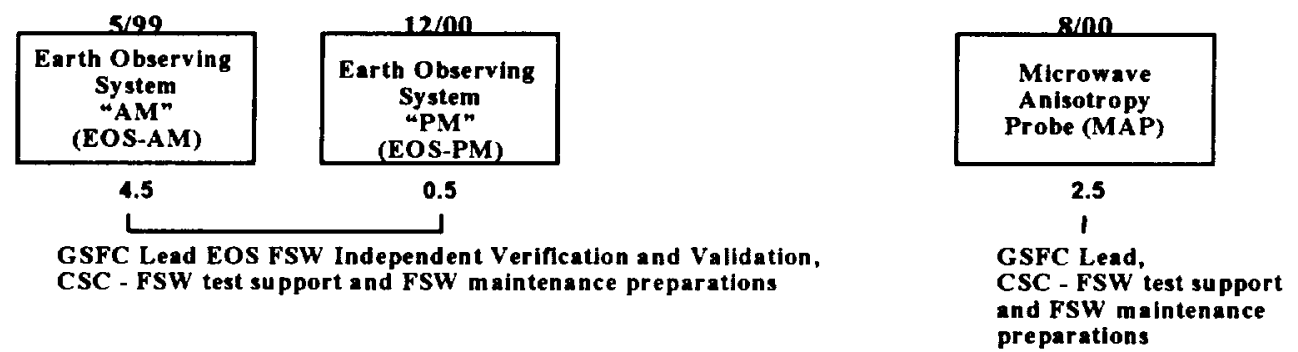

Figure 2 GSFC Missions Currently supported by the On-orbit FSW Team

Current FSW activity by on-orbit FSW staff includes:

- CGRO: Analog to digital gyro miscompare logic changes

- EUVE: Validations of testbed and tools for year 2000 rollover

- XTE: FSW estimation of solar array position using potentiometer data as contingency

- FAST: FSW changes to increase frequency of magnetometer data sampling for science correlation

- TRMM: Validation of onboard clock failure FSW contingency (based on pre-launch anomaly)

- TRACE: New onboard telemetry monitors

- SWAS: Early orbit operations support which includes new telemetry monitors

When one mission's need for FSW support decreases, assignments are realigned to support other missions with greater needs. The ability to move staff temporarily from one mission to another minimizes costs on each mission without immediate need for support, and it maximizes productivity on the "new" mission because experienced FSW talent is added to that team. This capability has proven to be a tremendously economical and low risk manner of supporting multiple spacecraft.

It is worth pointing out that the current missions, supported by this GSFC team, represent a variety of science objectives, orbital characteristics, flight data system architectures, flight hardware components, FSW languages, operating systems, complexities, and test environments. The software engineers on this team understand the overall nature of FSW in on-orbit environments, and they look at different implementations from a "systems" point of view. Members of this team take lessons learned from any mission and apply those lessons learned to all missions. Effective FSW maintenance procedures have been refined and documented based on similar FSW mission activities. The team was ISO-9000 certified in May 1998.

By housing the entire multi-mission FSW team within a single office area, sharing of experiences among all team members has been encouraged and successful. We believe that this co-location is critical to retaining the team over time. The long training period, the unending varieties of on-orbit experiences and the interesting variations of spacecraft and FSW characteristics creates common interests. This approach does not sacrifice the working interface with the FOT. Both groups routinely contact and visit each other to ask questions, get status, determine if interesting events are expected, etc.

Figure 3 represents the staffing levels of the CSC On-orbit FSW Team since its initial support to the Comptom Gamma Ray Observatory (CGRO) in 1988. Staffing for each new mission began well before launch, ramped up 
during the months preceding launch (typically to a peak of 2.5 to 5 people - see next paragraph), remained "high" for various numbers of months after launch, and essentially ramped down to a minimal level

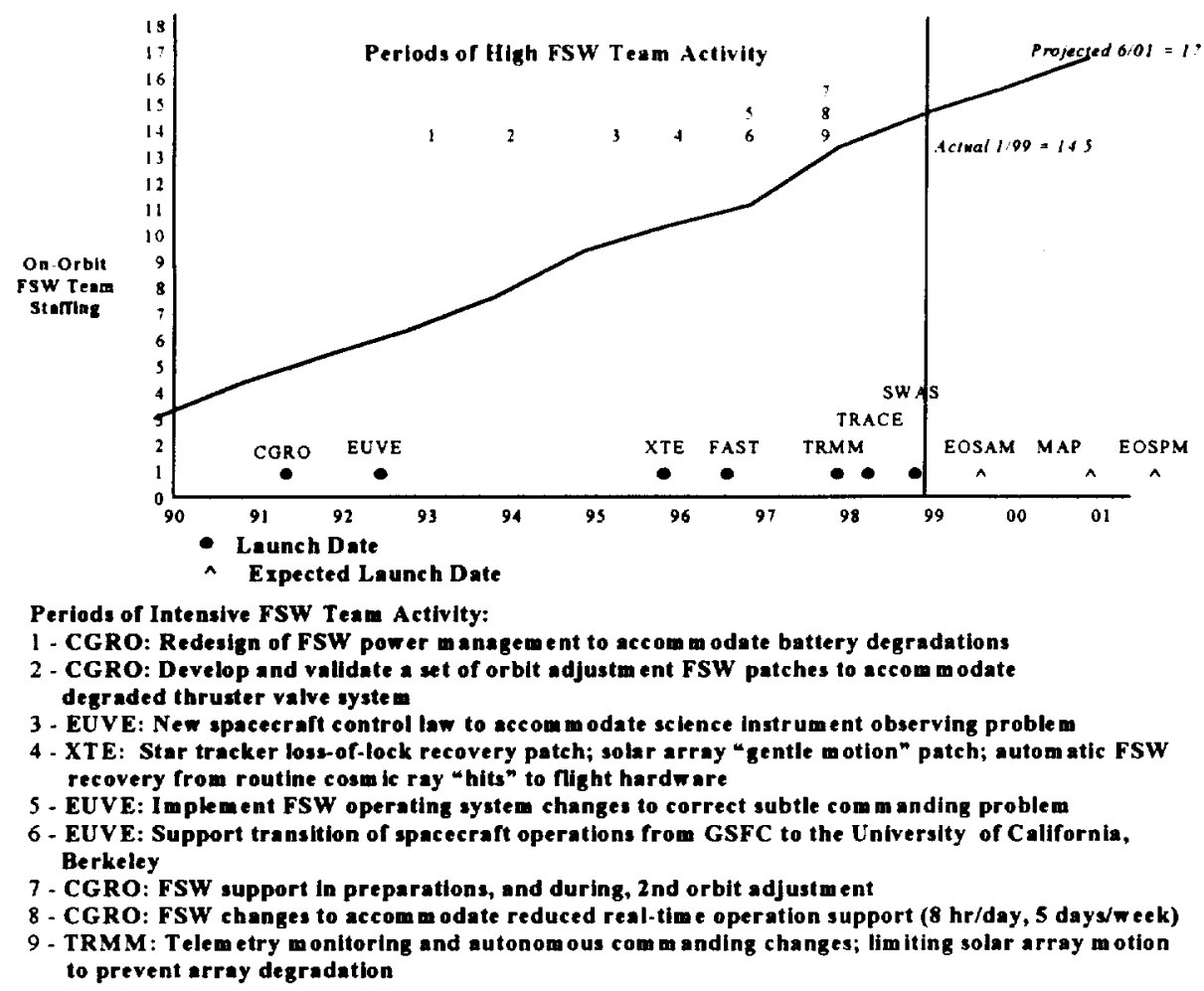

Figure 3 GSFC On-orbit FSW Center of Excellence Team Staffing over Time

after that - with staff increases during specific periods of approved FSW needs. From the launch dates shown in Figure 3, the slow increase over time of new mission responsibilities and staff is evident. The team has grown with careful assessment of staff strengths, commitments and mission situations. Because the training period is extensive and training does take time from more senior team members, it isn't practical to make commitments requiring a large increase in staff at one time. It appears that adding one or two new missions per year to the multi-mission team responsibilities provides a reasonable balance.

We can't emphasize enough the fact that the pre-launch staffing of FSW maintenance staff costs very little. The roles accomplished by team members are roles that would be necessary to staff with others. We find that FSW developers tend to want to move onto their next development assignment well before launch. If the maintenance people didn't prepare for on-orbit FSW maintenance, developers would need to do it. If the maintenance people didn't support the exhaustive mission simulations prior to launch, the developers or FOT would need to support more roles. This is true for system test roles, moving the FSW maintenance facility to its permanent on-orbit support location, certifying the on-orbit testbed, etc. The on-orbit team eliminates staff that would otherwise have to be funded.

\section{Establishing a Strong Multi-mission On-orbit FSW Center of Excellence}

Our experience indicates that it takes a combination of critical elements to assemble and retain a quality multimission on-orbit FSW team.

\subsection{Commit Early that a Separate On-orbit FSW Maintenance Team is Appropriate.}

Roles and responsibilities, of both the FSW team and the customers, need to be negotiated early in a spacecraft development effort in order to assure an effective transition to a specialized on-orbit FSW team. Multi-year staffing and costs should be estimated through the design life of the mission, and beyond if possible. The sources of funds for both FSW staff and for maintenance of system administration support, network support, commercial equipment 
maintenance, configuration control tools, etc., and the source of funds for maintenance of special-purpose testbed elements all need to be worked.

A prerequisite to taking on new FSW work is always the availability and fidelity of the FSW testbed to be provided to the maintenance team. Management of the on-orbit FSW team must be firm that a quality testbed must transition to the full authority of the on-orbit team. Sharing a testbed with a new development effort is not viable, and not having a testbed at all is not reasonable. Any scheme that does not include a high fidelity fully dynamic testbed for on-orbit support should be avoided. However, on many occasions we have shared the F.SW testbed with the FOT (during pre-launch simulations and post-launch operations procedure or database checkout). Since these two groups are both committed to the same mission and tend to have the same priorities, sharing has not been a problem to date.

GSFC has found that a brief written agreement between the FSW organization, the spacecraft development organization, the post-launch management organization and the science team lead (i.e., if different groups exist), is very effective toward assuring that commitments are clear.

\subsection{Carefully Select Personnel for the On-orbit FSW Team.}

Interviewing and selecting staff for on-orbit FSW maintenance is probably the most critical aspect to the success of the team. The work has tremendous responsibilities and at times can be very stressful. The strategy at CSC has been to select good software engineers who are enthusiastic about the space program. Insight into hardware electronics and interfaces is very useful since flight and testbed hardware can cause problems which the FSW specialist will need to resolve. The best candidates are confident software engineers who are more interested in the application of software than the immediate state of the art of software engineering. A love for, problem solving-rather than the fad of current programming languages or the most popular machines--has proven to be a common team characteristic. A considerable amount of time will be spent at the test facility creating tests, and validating FSW behavior, and the interview process should try to confirm that frequent FSW testing will not become too tiresome.

The combination of long training times, small staff and great responsibility means making mistakes in hiring can become very expensive in cost and mission support. It is seldom that one finds perfect candidates who have all, or most, of the emotional characteristics and the knowledge and skill levels to fit right in without significant training. A history showing a high level of commitment, maturity, flexibility, and initiative can indicate that the candidate will grow into the role and stay after the training period. The payback to the successful candidate is recognition within the user community, among the customers and within oneself that you have contributed to assuring science operations and extending the life of a spacecraft. Satisfying experience across the entire spectrum of spacecraft operations is another strong selling point.

\subsection{Select and Support the FSW Team Manager.}

The overall FSW Manager of the contractor team is a highly critical position. This is the person who establishes the talent mix and the work environment of the entire team. He/she must have the vision and appropriate technical insight that creates on-going enthusiasm for the work. This activity cannot be managed similarly to most project teams because there is no natural (foreseeable) ending of support for any mission and the demands on the FSW team can change dramatically at any time due to on-orbit events for any one mission. Team priorities tend to be flexible, and the manager has the responsibility to quickly adjust milestones and staff assignments to meet changing customer needs.

The success of an on-orbit FSW Center of Excellence may be directly related to the ease of sharing spacecraft, FSW and testbed issues among team members. As mentioned in 2.3, retention of a single office area should be a high priority for the team manager. Only when persons are assigned to pre-launch development activities, should members of the team be allowed to have desk space elsewhere.

A very high priority of the manager must be to ensure that individuals on the team do not stagnate in one area of involvement and lose interest in their work. New mission customers must be continually sought with reasonable phase-in periods to provide new opportunities for the team. Similarly, new team members must be continually brought onto the team to accommodate turnover of staff, and to provide teaching opportunities for experienced team members, who increase their skills when working with new persons. The manager must always ensure that a range of on-orbit FSW talent is available to contribute to any mission's on-orbit issues, and even when only minimal funding is available, the manager must always try to ensure that multiple people are trained for even the oldest missions.

\subsection{Staff up for FSW Maintenance Well Before Launch.}


Participation of intended FSW maintenance staff on the FSW development team and/or taking full responsibility for pre-launch FSW changes (under the guidance of the development staff) is ideal. Participation on a pre-launch FSW system test program seems to be a very good alternative, once the on-orbit team is established with several senior mentors to help with FSW-unique training. The more experience the on-orbit team has with development and test tools, test data, test procedures and results, the easier the transition to operations will be. Further, this involvement of maintenance personnel in a development effort brings an on-orbit systems perspective to the development team products. Early identification of FSW characteristics, which can minimize operations confusion, maximizes the chances that there will be time and inclination to modify development decisions for the sake of long-term operations and FSW maintenance

At least six months before launch, the FSW maintenance team needs to focus primarily on preparations for on-orbit FSW maintenance. The team will need to

- move the FSW testbed to its permanent on-orbit location (if necessary) and re-certify the FSW testbed

- study each aspect of the FSW requirements, design, code

- establish and validate the long term configuration control for both software and test products

- develop, validate and archive FSW regression tests

- understand the on-orbit FSW behavior

- develop and validate uplink procedures for flight data and code

- address all the documented lessons learned by the FSW team regarding launch-readiness.

FSW maintenance staff support of pre-launch mission simulations provides added familiarization with the FSW plus practice supporting the FOT. Active participation in launch and orbit insertion provides insight into early spacecraft performance which will help the FSW maintenance team understand later FSW on-orbit performance.

Once on-orbit the FSW maintenance team should work closely with the FOT to prepare, test and load changes to FSW parameters to tune the spacecraft for efficient long-term operations. If problems come up which require modifications to FSW (e.g., star tracker hardware loss-of-lock problems on the X-Ray Timing Explorer (XTE)), the on-orbit team is typically assigned the work with review from the development staff. When the spacecraft settles into long term operations (usually weeks to months after launch), the maintenance team is transitioned into the routine maintenance phase. Usually at this point some of the personnel are transitioned off to work on other spacecraft as determined by overall priorities.

\subsection{Provide Special Purpose FSW Training.}

It's important to recognize that an on-orbit FSW specialist requires training well beyond that of a FSW developer. FSW requirements, architecture, design and code are reviewed, and the FSW test system and support tools are used. In addition, the basic training includes highlights of mission characteristics, the spacecraft interfaces, timing characteristics, flight hardware features, attitude control subsystem issues, orbit determination processing, command/data handling applications; and other mission-unique capabilities related to FSW. On-orbit FSW specialists are primarily trained by each other exploiting the combined experience base of the team. A type of apprenticeship is used to allow new people to work on existing spacecraft with experienced people.

New team members are often requested to study different parts of the mission FSW and give tutorials to each other on the requirements, design, code, data, and tests. A strong internal tutorial process builds a cohesive, inquisitive team with knowledge about more aspects of the FSW than any member would have gotten on his own. When team members with experience on other missions are added in, the team develops insight into more than the spacecraft for which they are being trained, and recognition of the variety of FSW implementations makes the work continually interesting

A very rigid, risk mitigating FSW maintenance process has been established by the CSC FSW team. FSW maintenance standards and guidelines, refined by experiences on many missions, need to be explained in detail to new team members. All team members must understand "what" must be done, "how" it must be done, and "why" it should be done that way.

CSC periodically provides specialized training in spacecraft-related topics to increase team knowledge about how spacecraft operate, including courses in spacecraft design, attitude control, spacecraft dynamics, and spacecraft communications. Training in current software technologies is also provided. The new skills can be used to develop tools to help them to perform their job more easily while keeping up with the current technology.

CSC has found that it usually takes 6 to 9 months of training to bring someone with experience in software, spacecraft or operations to a level where he/she will be able to work without significant team support. 
3.6 Establish a Single Multi-mission On-orbit FSW Configuration Control Board (CCB) Chairperson. Independent of the FSW maintenance approach used, an on-orbit FSW CCB should be established for each mission. A CCB holds periodic meetings to discuss new work requests for the FSW team and to approve/disapprove, track and review all FSW-related activities. At GSFC, the CCB meetings for each mission supported by the FSW team, are chaired by a FSW expert from the GSFC FSW organization. Routine attendance by all FSW team members familiar with the mission and FOT personnel working that mission is required at any meeting. While always invited, original FSW developers, subsystem specialists, and science representatives tend to attend only when items of interest are to be discussed. It's important to emphasize that only work approved by the FSW CCB is authorized to be done by the FSW team. Other than on-orbit support, the team does not determine its workload prior to CCB approval of requests.

A FSW CCB Chairperson is typically responsible for obtaining, tracking and monitoring funds for FSW support of on-orbit mission. By ensuring that missions supported by the multi-mission FSW team are chaired by the same FSW expert, the Chairperson for all missions has responsibility for establishing the priorities among the entire set of missions supported by the team. He/she must have the insight to communicate technical issues, assess overall team capabilities at any time, and negotiate with individual mission operations and science teams to utilize the FSW maintenance team most effectively to each mission's advantage.

\subsection{Re-commit Funding for the FSW Team Yearly.}

Plan to review and update staff and funding commitments at least yearly. Retention of funds seems at times to be the most difficult of all tasks. In addition to funds for staffing the on-orbit FSW team, funds for test facility maintenance are necessary (see 3.1). It will help to have for discussion with the customer, detailed documentation of special FOT support, on-orbit FSW changes, testbed maintenance support, training, etc. which has taken place since the team was put into place. Data related to the causes of staff increases and decreases should be maintained. Routine contact with the customers even during slow periods should be a priority.

Even for aging missions with no current FSW activity, a minimum amount of funding needs to be available in order to maintain the FSW environment and to train staff, if necessary, for that mission. A commitment of reasonable funds permits the FSW manager to use his/her judgement as to what "background" tasks need to be accomplished and whether adequate team resources are trained for the mission. A FSW policy regarding this at GSFC is that a minimum of one man-year of funds should be provided for each mission. Unspent funds routinely revert back to the customer at the end of the year. The fact that funds are clearly not spent unless there is a need has created an environment of trust that remains from one year to the next while assuring that the FSW team can meet it's needs if necessary.

\section{Conclusion}

GSFC has learned from experience that a multi-mission on-orbit FSW maintenance team works. It is not an easy team to establish and maintain but, with reasonable planning, it can be done, and the payoff is significant. All customers seem to appreciate the level of talent we are able to provide to their mission's FSW needs. Team members seem to enjoy the continual variety of spacecraft FSW issues and problems to solve. The overall results of growing the team over a 10 year period seem to indicate that mission risks are minimized for a very low cost investment in training and personnel retention. Team productivity has increased with the sharing of lessons learned. A strong technical support team has been developed which can be focused on any mission. We believe that science return has been maximized by consistent availability of mission critical FSW expertise during operations.

We have also learned some techniques that help achieve our goals. Highly routine communications with FSW customers is necessary to make the contributions of the team apparent and to assure reasonable funds. Tracking of small tasks by the team as well as large more visible tasks has proven to be very useful in demonstrating the contributions of the team. A monthly summary of the combined accomplishments and issues (e.g., technical impacts and funds) of all missions is now being distributed to customers, FOTs, managers, etc. in order to represent the full activities of the team to all customers.

To a limited extent we have been successful at assigning on-orbit FSW maintenance personnel to new spacecraft development activities. Additional value of this team capability will occur when on-orbit maintenance staff routinely participates in FSW development team activities. This "closed loop" sharing of lessons learned has the strong potential to create even more efficiencies and performance in the end-to-end FSW product. 
Acknowledgements: Special recognition to Mike Oben of GSFC for serving tirelessly as the overall government lead and CCB Chairperson of the multi-mission contractor FSW Center of Excellence team; and to Lou Hallock of GSFC for excellent suggestions during development of this paper.

\section{References}

Maldari,P.,Jorgensen,C., and Baudoux,D., "On-board Software Maintenance - an Operational Task", International Symposium on On-Board Real-Time Software. ESTEC, Noordwijk, The Netherlands, November 1315,1995 . 